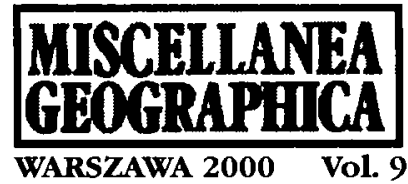

\author{
Jolanta Korycka-Skorupa
}

\title{
FROM DATA TO CARTOGRAPHIC PRESENTATION FORMS
}

Data constitute the starting point for any cartographic presentation. Elaboration of a map requires prior acquisition of data. This can take place through measurement of the phenomenon, which is to be shown, or by making use of the data sets already existing, like statistical yearbooks or databases.

Having data, the author of the map must decide how to present them. Decision therefore must be made as to what graphical form should be used in order for the users of the map to correctly interpret it. Editor of the map disposes then of a broad scope of graphical means, which are mostly referred to in cartography as methods of cartographic presentation.

When speaking of the method of cartographic presentation we usually mean the map already elaborated, the graphical effect. Yet, in methodology, the term method is used not to design the effect, but the way of proceeding. A better designation for the end effect, i.e. for the map that is already executed, is the form of presentation. This term will be used in the present report as referring to the ultimate image, the concrete graphical solution.

The process of transition from the statistical data to the forms of cartographic presentation is the essence of cartographic methodology. It is therefore worthwhile to consider the essence of the form of cartographic presentation.

It is known that this is a certain graphical image ("external aspect"). In order to obtain it, it is necessary to have data and to carry out two operations. Thus, the presentation form is composed of:

- two kinds of data: the initial data (in the form, in which we acquire them), and the transformed data (adapted to the requirements of the concrete graphical presentation), and

- two processes: transformation and visualisation (see Fig. 1).

At the stage of transformation we adapt the data to the requirements of presentation. We can therefore perform certain operations connected with:

- level of measurement, at which the data have been expressed (here I assume three levels: qualitative, ordered, and quantitative);

- data reference sets (points, lines, planes);

- nature of data (relative, absolute);

- way of treating data (continuous, discrete). 


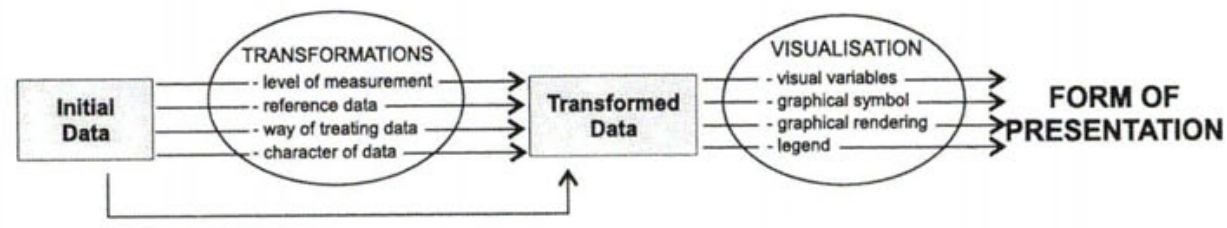

Fig. 1. From data to cartographic presentation forms.

The features listed characterise the initial data. They are ascribed to the data already at the instance the author of the map starts to edit it. Yet, the author can adapt the data acquired to the needs of the map being elaborated. Hence, transformation constitutes the stage at which it is possible to:

- reduce the level of measurement of data;

- change the nature or the way of expressing data (e.g. by transforming the absolute data into the relative ones);

- discretise data by defining appropriate classes.

The data that we are going to present may be expressed at the different measurement levels (Chang, 1978; Robinson, 1995). For the purposes of this report it seems sufficient to deal with just three general levels: qualitative, ordered, and quantitative. The different measurement levels, at which the data presented are expressed, entail application of different graphical solutions on the map. The transformed data, prepared for presentation, have then to be visualised. Rendering them in a concrete graphical form will make us deal with a definite presentation form. The cartographic presentation form is therefore the effect of a logical combination of the features mentioned before. Visualisation requires that the author of the map:

- chose the visual variables, which will be used in presentation;

- chose the graphical sign related by its very nature to the reference set (point, line, plane);

- made decision on the graphical treatment of the data (continuous or discrete graphical presentation);

- elaborated a correct legend.

For purposes of this report I adopted the visual variables proposed by J. Bertin (1983). These variables include: shape, size, texture, value (understood as the proportion of the white to the black), colour, orientation, location (situating the object by specification of two coordinate values $-\mathrm{x}, \mathrm{y}$ ).

The variable of location, mentioned by J. Bertin, differs distinctly from the other ones. Each object on the map can be namely assigned a definite position (expressed through the variable of location) and some other feature (expressed through another variable). Location is therefore a supreme variable with respect to the other ones. It is also worthwhile to unequivocally define colour, as listed also by J. Bertin. It is in fact one of the three features of the colour, referred to as hue (Robinson, 1995).

The classification of visual variables provided by J. Bertin was considered 
by the cartographers as useful, but subject to discussion. During the last dozen or so years attempts have been undertaken of modifying the variables, of adapting them to the needs of cartography, and to the new technological conditions (MacEachren, 1995). The foundation for the considerations concerning the correctness of application of the visual variables at the particular measurement levels is, however, constituted by the concept of J. Bertin, as the most popular and most often cited. In the study here reported the variable of location will not be accounted for. It constitutes the basic attribute of any map, and so, the consideration of the correctness of its application at the particular measurement levels seems to be unjustified.

The here presented scheme of transition from data to cartographic presentation forms seems to be quite simple and logical. Is it, though, correct? Does it exhaust all the aspects of the issue considered? Can any form of cartographic presentation be unambiguously derived from this scheme?

Publications from the domain of cartography devote ample space to the study of particular forms of cartographic presentation. Various authors provide different classifications of the presentation forms.

The most popular among the Polish cartographers is the classification of the presentation forms proposed by L. Ratajski (1989). The fundamental criterion in the establishment of this classification was the distinction of possibility of having quantitative and qualitative characteristics. Thus, L. Ratajski distinguished seven basic presentation forms: three qualitative (reaches, signatures, and the chorochromatic method), and four quantitative (choropleth map, map of graduated symbols, isarythmic map, and dot map). This classification was based upon the presentation forms, which had been distinguished before by A.H. Robinson (1953). Among the forms presenting quantitative data there are also the flow lines, which correspond in Polish terminology to map of linear graduated symbols, as well as the dasymetric map, being a special case of the choropleth map.

K.A. Salichtchev (1982) distinguished even more forms of presentation, namely 11 . Side by side with the forms mentioned before, his classification included also: quantitative signatures, movement symbols, the method of localised diagrams, and the method of quantitative background.

The scheme of transition from data to cartographic presentation forms, proposed here, and the attempt of connecting the measurement levels and the visual signs and variables, lead to appearance of a number of potential of graphical solutions - cartographic presentation forms. In further considerations I will try to establish connections between the features listed. Such an analysis was conducted by J. Bertin (1983) with respect to visual variables and measurement levels. According to him, in presentation of data at each particular level, an appropriate visual variable ought to be applied, as resulting from the perception capacities of the user of the map (Fig. 2). I would like to go a step further, in order to reach the phase of the forms of presentation. The respective analysis, though, does not account for the reference sets of the phenomena and the manner of their appearance, since 


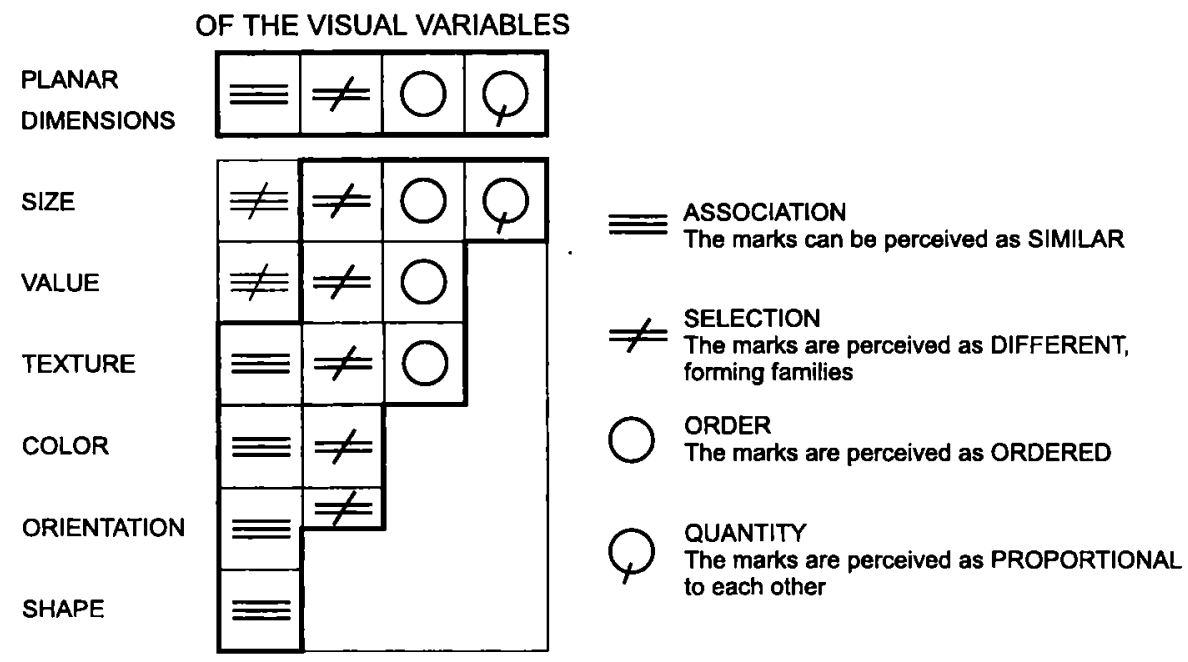

Fig. 2. Properties of the individual visual variables according to J. Bertin (1983).

these properties of phenomena do not always find reflection in the graphical image (a point-wise phenomenon can be shown through a surface, while a spatial phenomenon can be shown in a discrete manner). Likewise, the manner of expressing data (relative, absolute) is not accounted for, because it is only in the case of a choropleth map that it decides of the cartographic presentation form applied.

The attempt of linking the graphical sign, the measurement level, and the way of expressing data is illustrated in the tabular form (Figs. 3, 4 and 5). Each graphical solution, each entry to the table was subject to logical and methodological verification (has this presentation of contents sense? is it methodologically correct?), and to verification of application of a given solution (is this sort of solution used on the maps?). These solutions, which are methodologically correct and widely applied have been given names.

\section{POINT SYMBOLS}

\section{1. at the qualitative level}

Presentation of qualitative data cannot be made with the use of signs whose value (brightness) and size change. Such solutions have to be rejected, since they allow reading from the map more than the map actually contains. If the signs representing quality (like "shop", "library", "museum") are differentiated as to their size, we are motivated to look for the "quantitative" differences between the objects, which at this level differ among themselves in just a qualitative manner. A dfferentiation of the signs mentioned with value may cause, on the other hand, that we will be trying to order the objects, which by the very nature of the measurement level cannot be or- 
dered. Qualitative data can be shown with the help of all the remaining visual variables. Their application, though, differs, because they vary as to their legibility. Hue and shape are applied more often, because it is easier to distinguish them than different textures and orientations. In Polish cartographic terminology all such solutions are referred to as point symbols.

\section{2. at the ordered level}

At the level of the ordered measurements the best solution is to apply value (brightness). Differentiation of the standardised signs (like circles or squares) with the help of value makes it possible to sequence them, but does not allow estimation of the associated magnitudes. Due to application of value at this level there is no possibility of excess interpretation of the map - one cannot read "too much" from it. Such a graphical solution is methodologically correct and is being applied in practice. It can be referred to as the point ordered choropleth map.

It is also admissible to use as representations of data at the level of the ordered measurements the signs differentiated as to their size. It seems that this solution is being applied the most frequently, although it is not as correct as application of value (since it may entail an over-interpretation of data, as it is possible in this case to determine the differences between the particular objects). This kind of solution is called the point ordered diagram map.

The remaining visual variables (hue, size, texture and orientation) do not possess the ordering properties. Yet, one can find maps, in which ordering is expressed through application of hue (e.g. the hypsometric scale) or texture. The hypsometric scale is a specific setting of hues, which is additionally ordered with value. Thus, it is a kind of exception. No combination of hues (without a simultaneous application of value) gives the impression of ordering. A similar situation exists with respect to texture; although objects could in principle be "ordered" (e.g. in accordance to the magnitude of granulation), we would not be able to tell the direction of this ordering - which extreme of the scale constitutes the end, and which is the beginning.

\section{3. at the quantitative level}

Similarly as at the ordered level, presentation of data at the quantitative level cannot be made with the use of such variables as hue, shape, texture and orientation. Application of these variables does not allow to quantitatively differentiate objects, that is - to estimate the respective magnitudes.

The best solution is to apply differentiated size. Showing the differences of magnitude between the objects through differentiation of the sign size is a commonly applied solution, designated as the point diagram map (continuous or discrete, depending upon the way the variables are treated). In a particular case, when the size of a sign does not change in proportion to the differences of magnitude between the objects, we deal with the quan- 
titative point symbols. It is necessary, though, to preserve an adequate optical weight of individual signs. The effect of optical weight is often achieved through additional manipulation on the value of the sign. This solution was not accounted for in Fig. 2, because it is not an example of application of just one variable, but of a combination of several visual variables.

It is equally common to apply value on the quantitative level, through filling of a standard shape and size of a point sign with varying value. This solution is not entirely correct (it is difficult to assess the differences of quantity shown through varying value), but often applied. In analogy to the ordered level we can call this presentation form point choropleth map. This presentation form differs from the popular choropleth map by the applied graphical sign and the reference used (the choropleth map in the usual understanding is an areal map, while in this case we deal with points).

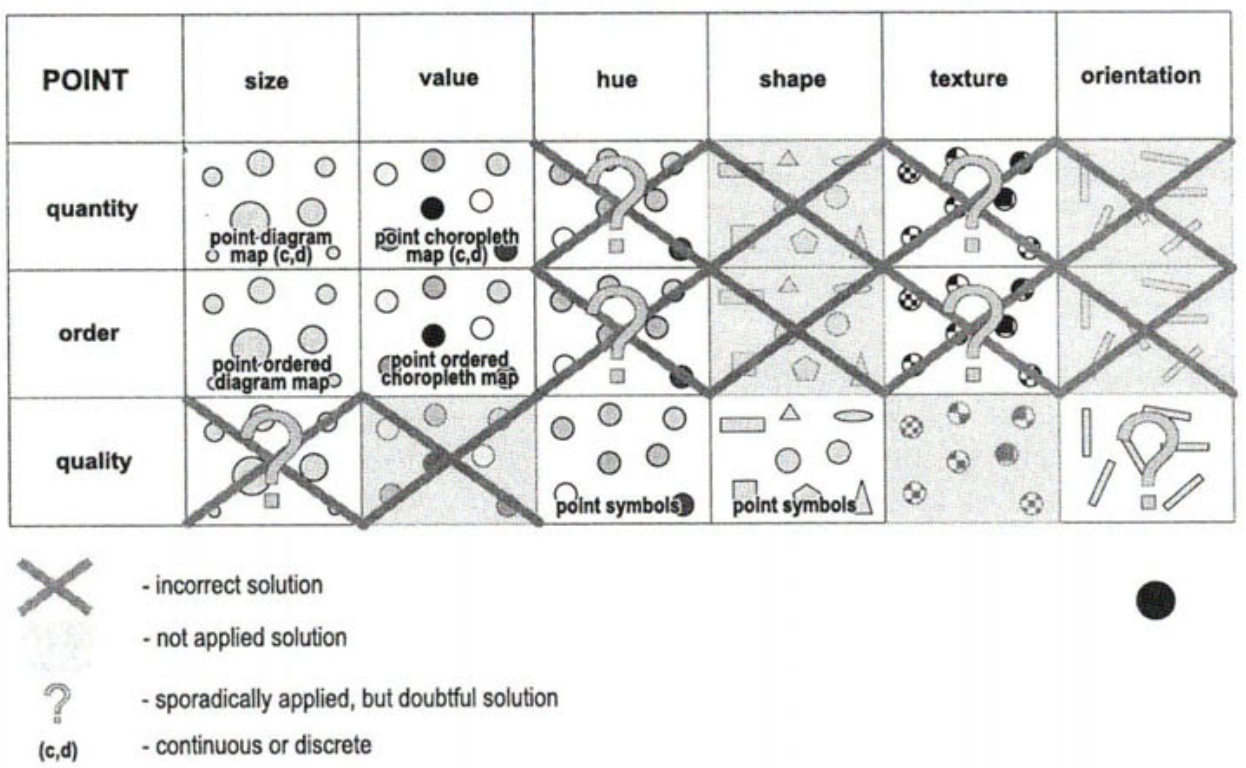

Fig. 3. Visual variables and measurement levels in application to point symbols.

Another commonly applied graphical solution is the choropleth map in diagrams, consisting in the simultaneous application of two visual variables (size and value). This approach is most often used to present two different characteristics of a phenomenon (e.g. the size of diagrams may indicate the population number, while their value - the share of one of the age groups in total population number). It is possible, though, to find maps in which just one phenomenon is thus presented, with the use of two and not only one - variables. This solution was not taken into consideration in Fig. 2, since it requires application of two visual variables simultaneously. 


\section{LINE SYMBOLS (Fig. 4)}

\section{1. at the qualitative level}

A line symbol, meant to represent qualitative data, can be differentiated with the hue, shape, texture, or orientation. Such a sign can be used to show both the phenomena of linear reference (these are the line symbols), and the ones of planar reference (in the form of boundary lines).

As mentioned already before, the variables of size and value are not appropriate for presentation at the qualitative level.

\section{2. at the ordered level}

Like in the case of point symbols, order relations can be represented through changing value. Such a visual solution is encountered on the maps, although until now it has not been named. Keeping to the previous principles this solution ought to be called linear ordered choropleth map.

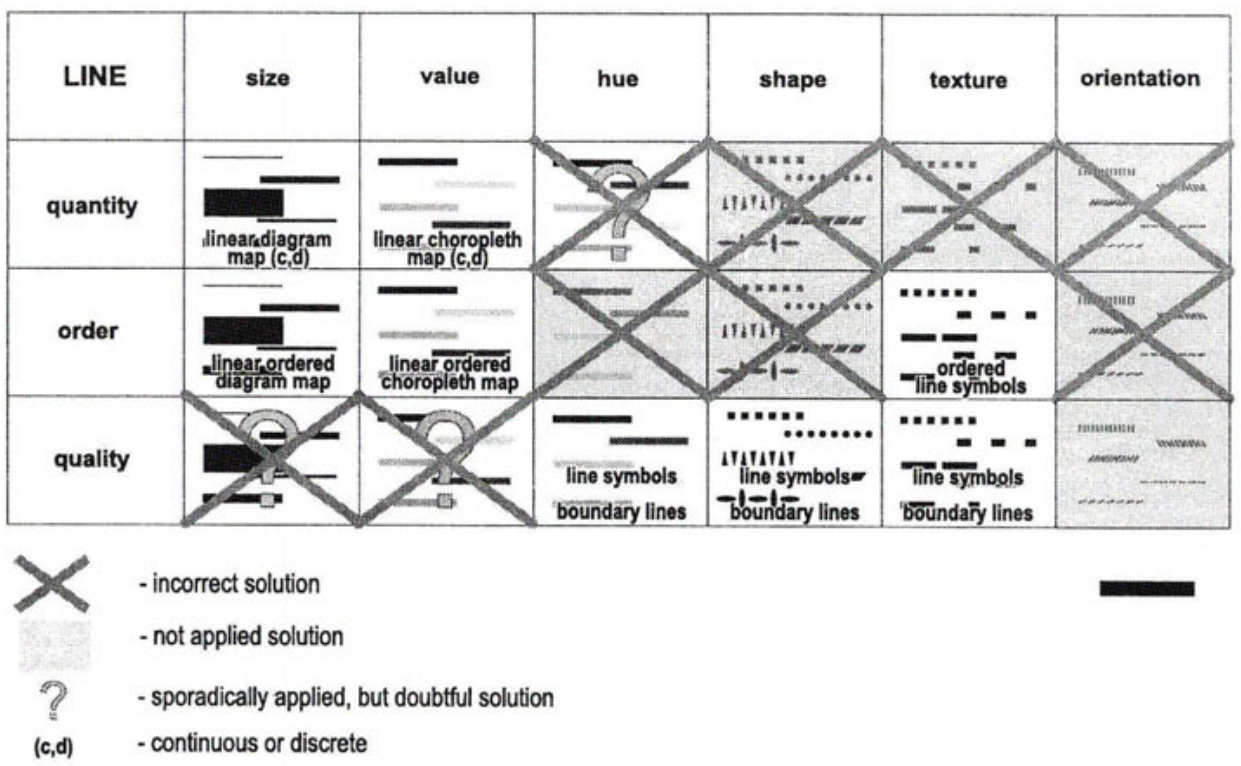

Fig. 4. Visual variables and measurement levels in application to line symbols.

When the data are expressed on the ordered level, and shown by a line of a variable dimension, then we deal with the linear ordered diagram map.

From among the remaining four visual variables only texture is being applied to line symbols. Here, though, it is not texture in the "pure form", insofar as it is sometimes additionally differentiated by the optical weight of a sign. Symbols with finer granulation appear as more delicate than the ones with coarser granulation. One can therefore say that this is a combi- 
nation of texture and value. An example of such an application is demonstration on the map of different ranks of administrative boundaries (of communes, counties, provinces). Since this is not a simple application of a visual variable, it was not accounted for in Fig. 3.

\section{3. at the quantitative level}

With respect to the line symbols it seems appropriate to use on the quantitative level only two visual variables: size and value. By changing the dimension (thickness) of the line we can obtain the linear diagram map (continuous or discrete).

Less popular - and less correct - is to apply value. For the sake of consistency with the earlier considerations such a graphical solution ought to be called linear choropleth map.

\section{AREA SYMBOLS (Fig. 5)}

\section{1. at the qualitative level}

Similarly as in the preceding cases, area symbols can be used to represent quality, with the filling of the area differentiated by hue, shape, texture, or orientation. Such visual solutions are known to cartography as the chorochromatic (area) method. In the same manner we can show data with the help of boundary areas.

At this measurement level it is incorrect to apply sizes and values, since they bring in the order-implying, or even quantitative, contents.

\section{2. at the ordered level}

For reasons mentioned already before two visual variables can be applied on this level. Order relations are, naturally, best rendered by differentiation of value. This kind of graphical solution can be called the area ordered choropleth map. In the same case we can also speak of the ordered dasymetric map, which differs from the choropleth map solely by a different approach to the reference set.

J. Bertin (1983) proposed to apply the variable of size to the filling pattern of the area symbols. This kind of graphical solution is called ordered Bertin's choropleth map.

\section{3. at the quantitative level}

Differentiation of area symbols by the size of the pattern, which fills it, is known in cartography as the Bertin's choropleth map. Until now only a certain specific kind of choropleth map was referred to in this manner. It is clear, though, that this simply is a definite type of graphical solution.

Value is the second visual variable, which can be applied on the quantitative level (although, as we know from our previous considerations, this is not entirely correct). We obtain thereby the very popular in cartography 
area choropleth map (continuous or discrete), or its equally popular variation, the dasymetric map.

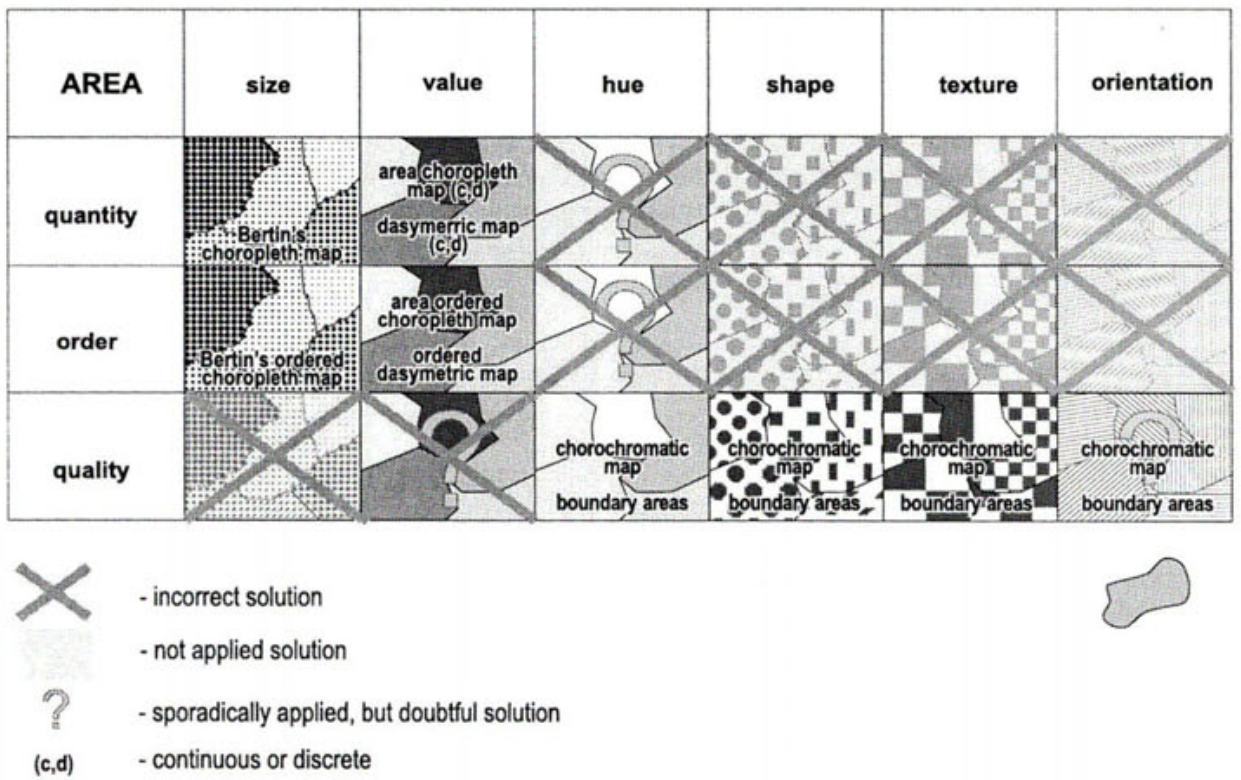

Fig. 5. Visual variables and measurement levels in application to area symbols.

Among seven cartographic presentation forms commonly known to and applied in cartography (Ratajski, 1973, 1989) there are two, which miss from the considerations here contained: isoline and dot maps. These two forms of presentations differ distinctly from the remaining ones. The fundamental graphical means is constituted in these forms by a single line or one dot (frquently black). A change in the magnitude of a phenomenon is reflected through a change in density (multiplicity) of signs (lines or dots), and not through application of one of the visual variables. It is only a definite set of symbols, together with relations among them in terms of their mutual disposition, that make it possible to read out some information from such an image. Both of these forms of presentation speak to us through more or less dense sets of symbols, that is - through optical weight and value of the image. Distribution of the individual symbols is not without significance, either. In these particular forms of presentation a special significance is gained by the visual variable of location, mentioned by J. Bertin. Thus, these forms of presentation belong to a completely different category. The system of visual variables proposed by J. Bertin (1983) does not offer any single variable or a simple combination of variables, which would allow describing these two forms of presentation.

The scheme of transition from the statistical data to the presentation forms makes it possible to track many of the graphical solutions. On the 
basis of such an analysis one can perceive certain relations between the measurement levels and the visual variables. Thus, size is the variable which functions well at the quantitative level, while value - at the ordered level. Both of these variables are, however, commonly used on each of these two levels. The remaining variables find application on the qualitative level. When assessing individual visual solutions we can distinguish a whole series of the cartographic presentation forms. Many of them exist in cartography, although they are not accounted for in the classification. This may perhaps be a good reason for attempting a change of classification, so as to account for all the feasible, correct, and applied solutions.

\section{REFERENCES}

B ertin J., 1983, Semiology of Graphics: Diagrams, Networks, Maps, The University of Wisconsin Press, Madison.

Ch ang K.-T., 1978, Measurement Scales in Cartography, The American Cartographer, $5,3,57-64$.

MacE ach ren A.M., 1995, How Maps Work (Representation, Visualisation and Design), The Guilford Press, New York-London.

Ratajski L., 1973 ( $1^{\text {st }}$ edition), 1989 ( $2^{\text {nd }}$ edition), Metodyka kartografii spotecznogospodarczej [Methodology of socio-economic cartography], PPWK, War-szawa.

Robins on A.H., 1953, Elements of Cartography, New York-London ( $1^{\text {st }}$ edition).

Robinson A.H., Morrison J.L., Muehrcke Ph.C., Klimerling A.J., Guptil S.C., 1995, Elements of Cartography, New York ( $6^{\text {th }}$ edition).

S a li ch t ch e v K.A., 1982, Kartovedenie [Cartography]. Izdatel'stvo Moskovskogo Universiteta, Moskva. 